\title{
Let us stop throwing out the baby with the bathwater: towards better analysis of longitudinal injury data
}

\author{
Caroline F Finch, ${ }^{1}$ Stephen W Marshall ${ }^{2}$
}

Sports injury prevention is a priority area in $B J S M^{1}$ and recent commentaries have stressed the need to consider relationships between sports injuries in longitudinal data sets. ${ }^{2} 3$ Players can sustain none, one, or more than one injury over a season of follow-up. Subsequent injuries are statistically related to prior injuries because they occur in the same person. This is true even when the two injuries are clinically distinct. $^{2} 45$

It is always important to collect, analyse and report data on subsequent injuries in injury incidence studies. Figure 1 shows a hypothetical cohort of five players followed over one season of 10-week duration. This example assumes that all players are injury-free at the start of the season and addresses acute onset injuries

\footnotetext{
${ }^{1}$ Australian Centre for Research into Injury in Sport and its Prevention (ACRISP), Federation University Australia, Ballarat, Victoria, Australia; ${ }^{2}$ njury Prevention Research Center, University of North Carolina at Chapel Hill, Chapel Hill, North Carolina, USA

Correspondence to Professor Caroline F Finch, Australian Centre for Research into Injury in Sport and its Prevention (ACRISP), Federation University Australia, SMB Campus, PO Box 663, Ballarat, VIC 3353, Australia; c.finch@federation.edu.au
}

rather than those due to repetitive microtrauma. The figure contains considerable information on each player: how long they were followed up; the number of injuries they sustained; how long before they sustained their first injury; and how long after any injury it took before they sustained their next one. Open circles indicate points at which players were injured, and stars indicate when follow-up was censored (eg, player 2 was only followed up for 7-weeks for non-injury reasons). A player does not accrue time at risk when he/she is unable to participate. For example, all of player 3's injuries result in time loss (TL) from the sport for healing and rehabilitation. None of player 5's injuries, on the other hand, result in any $\mathrm{TL}$; these are known as non-TL injuries.

\section{THE PROBLEM}

Longitudinal sports injury data are often analysed in one of the three ways:

- A risk,

- A rate,

- Time to first injury (hazard).

All three approaches have profound limitations. $^{36}$
Some studies report injury incidence in terms of the number of injured players divided by the number of players on the team at pre-season (far right hand side, figure 2). This is the average probability of injury, which is a risk. A risk answers a question often voiced by players and/or their families at pre-season: What is the probability that I will be hurt this season? To compute a one-season risk, the data for each player is reduced to a binary outcome: yes-sustained $\geq 1$ injury, and no-remained injury free. Such analyses ignore how many injuries people sustain, as well as ignore the timing between subsequent injuries. In our figure, players 1 and 3 contribute the same information to a risk, despite of the fact that player 3 has three injuries and player 1 has one injury.

An alternative approach is the injury rate. The rate (or 'incidence density') is the number of injuries divided by the total person-time at risk (far right hand side, figure 3). Scientists use rates because, unlike risks, they use more of the injury information and account for variation in follow-up between respondents. However, rate does not have an obvious interpretation for nonscientists. A problem with rates is that the measure still ignores the length of time between injuries and inherently assumes that multiple injuries to the same person were unrelated. Thus, three non-TL injuries to three different players followed for 3 weeks each yields the same rate as three non-TL injuries to the same player followed for 9 weeks. However, these are two different situations from clinical and resource allocation standpoints. ${ }^{7}$ 


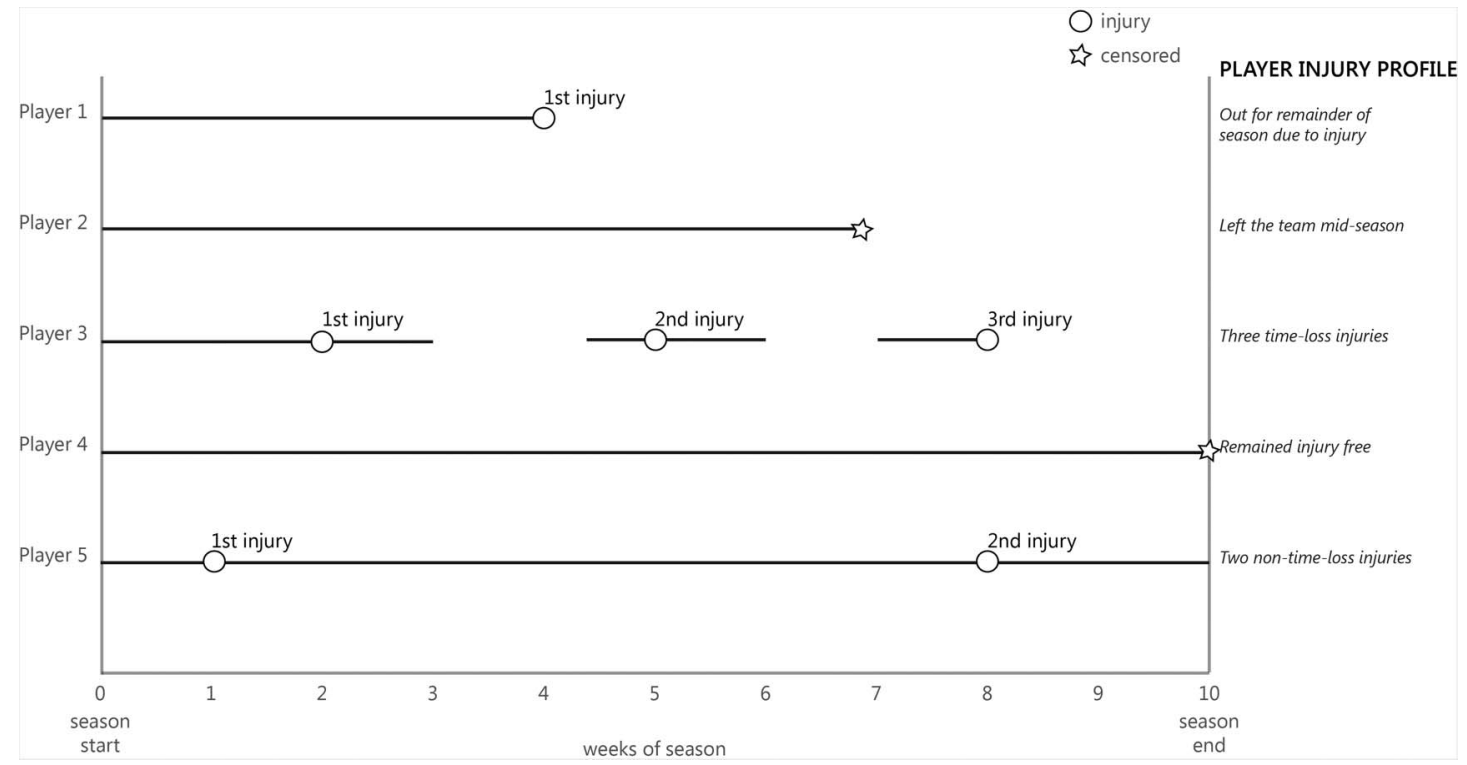

Figure 1 Hypothetical prospectively collected injury data.

The third approach is to use simple survival analysis to compute time to first injury (bold lines, figure 4). This quantity is known as initial hazard and it is equivalent to a rates analysis in which all respondents are censored after their first injury. But by limiting analysis to time to first injury only, this approach also excludes information about subsequent injuries. Thus data on only one injury is included for player 2 and data on two subsequent injuries is excluded from the player 3's injury profile.

\section{The solution}

Appropriate survival analysis methods are now available that make full use of all longitudinal sports injury data (figure 5). These use essentially the same model as simple survival with the modification that all time intervals are included: to first injury, between all subsequent injuries and through to the end of follow-up. When injuries are coded as index or subsequent injuries according to a classification such as the subsequent injury categorisation (SIC) model, ${ }^{2}$ relationships between injuries can be determined and analysed. Statistical techniques for longitudinal data sets incorporating all injuries and the intervals between them are relatively simple to implement in most statistical software and often merely comprise applying the standard survival model to a restructured data $\operatorname{set}^{8}$ or extensions to the usual Cox regression model. ${ }^{3}$

\section{SUMMARY}

Significant time and resources are expended collecting high-quality longitudinal injury data. However, most data analyses from these studies do not adequately address repeated injury events on the same athlete, and therefore squander useful data. More efficient analysis models are described in the statistical literature but, regrettably, are uncommon in sports medicine. ${ }^{3}$ Importantly, the quality of the scientific evidence needed to underpin clinical

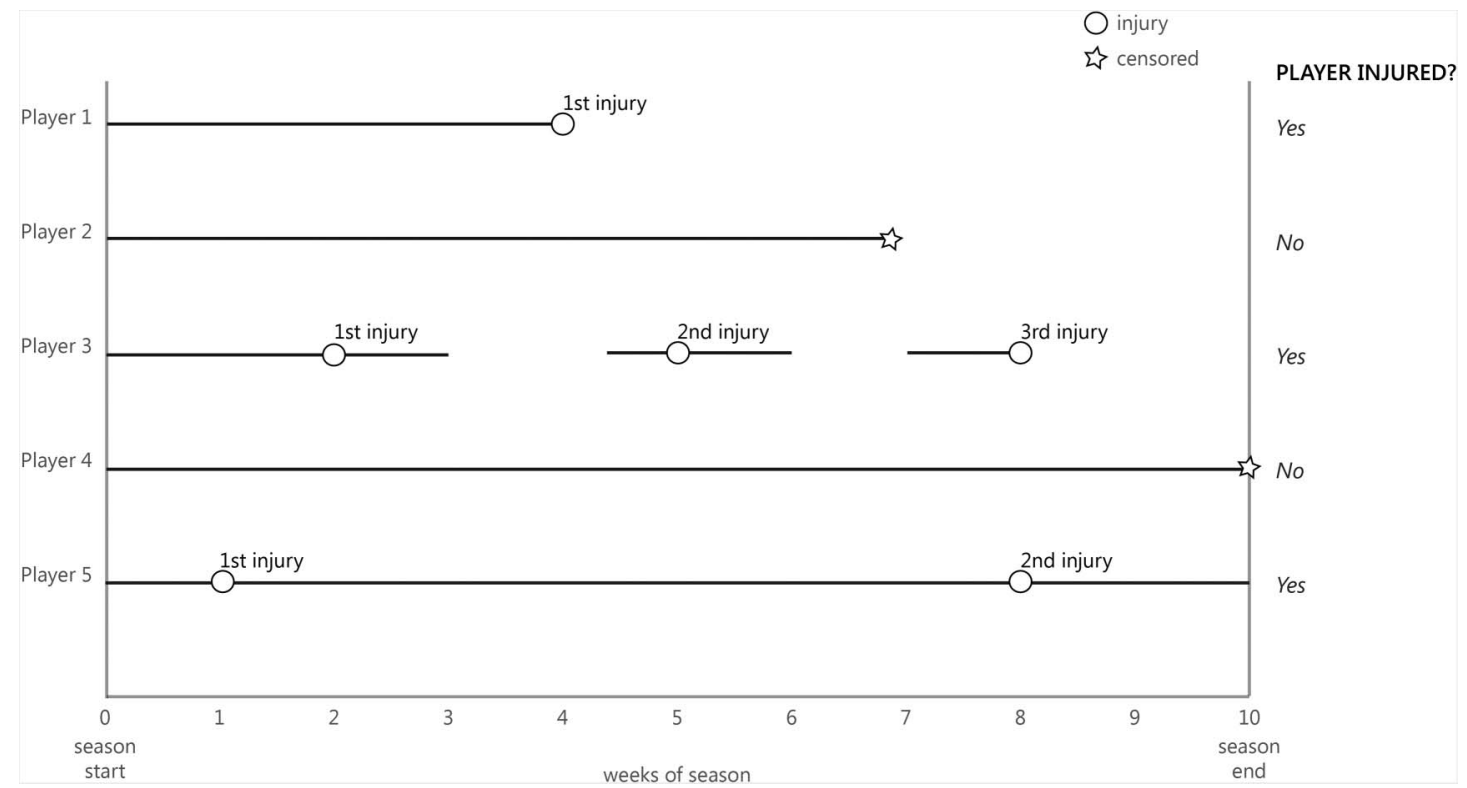

Figure 2 Common approach to reporting injury incidence in terms of injured athletes. 


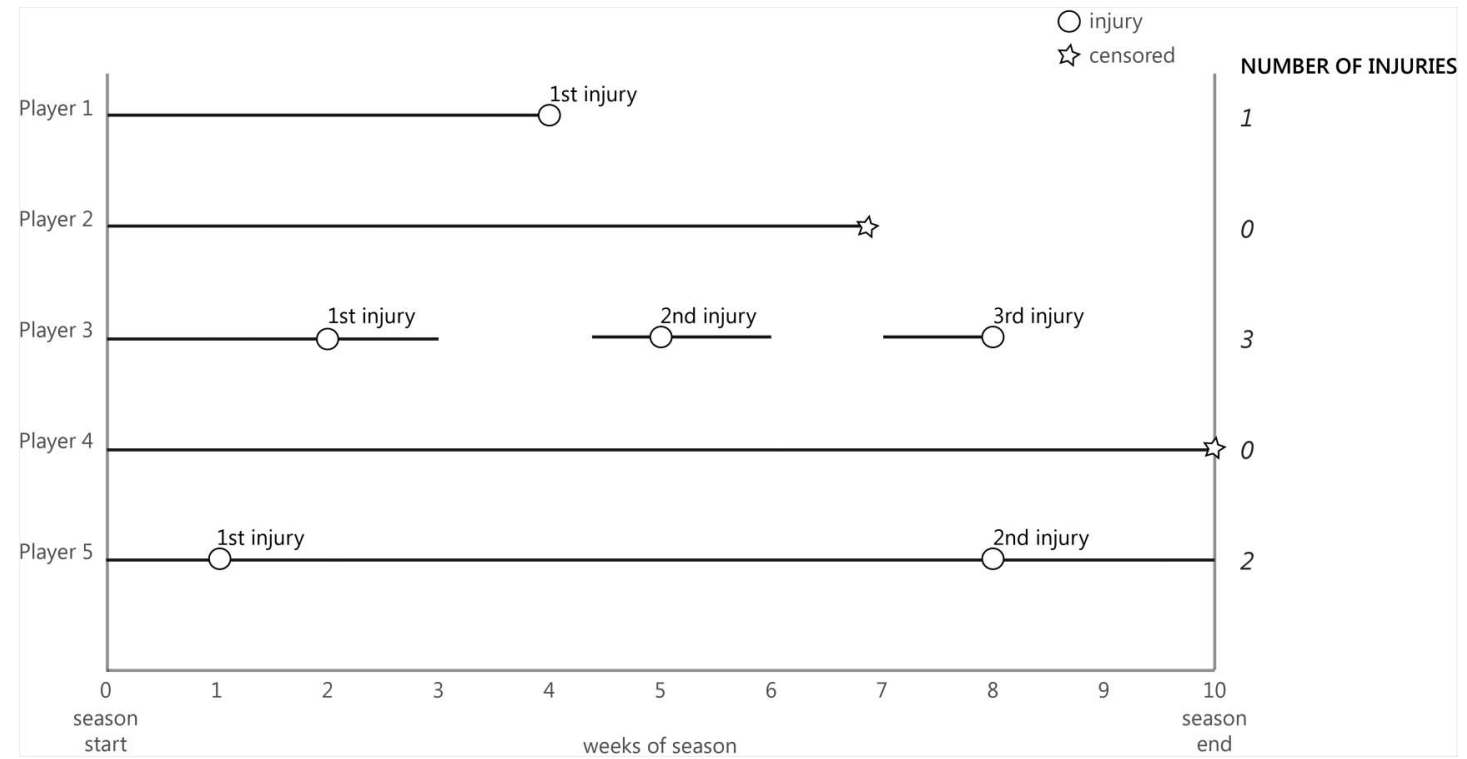

Figure 3 Common approach to reporting injury incidence in terms of injury counts.

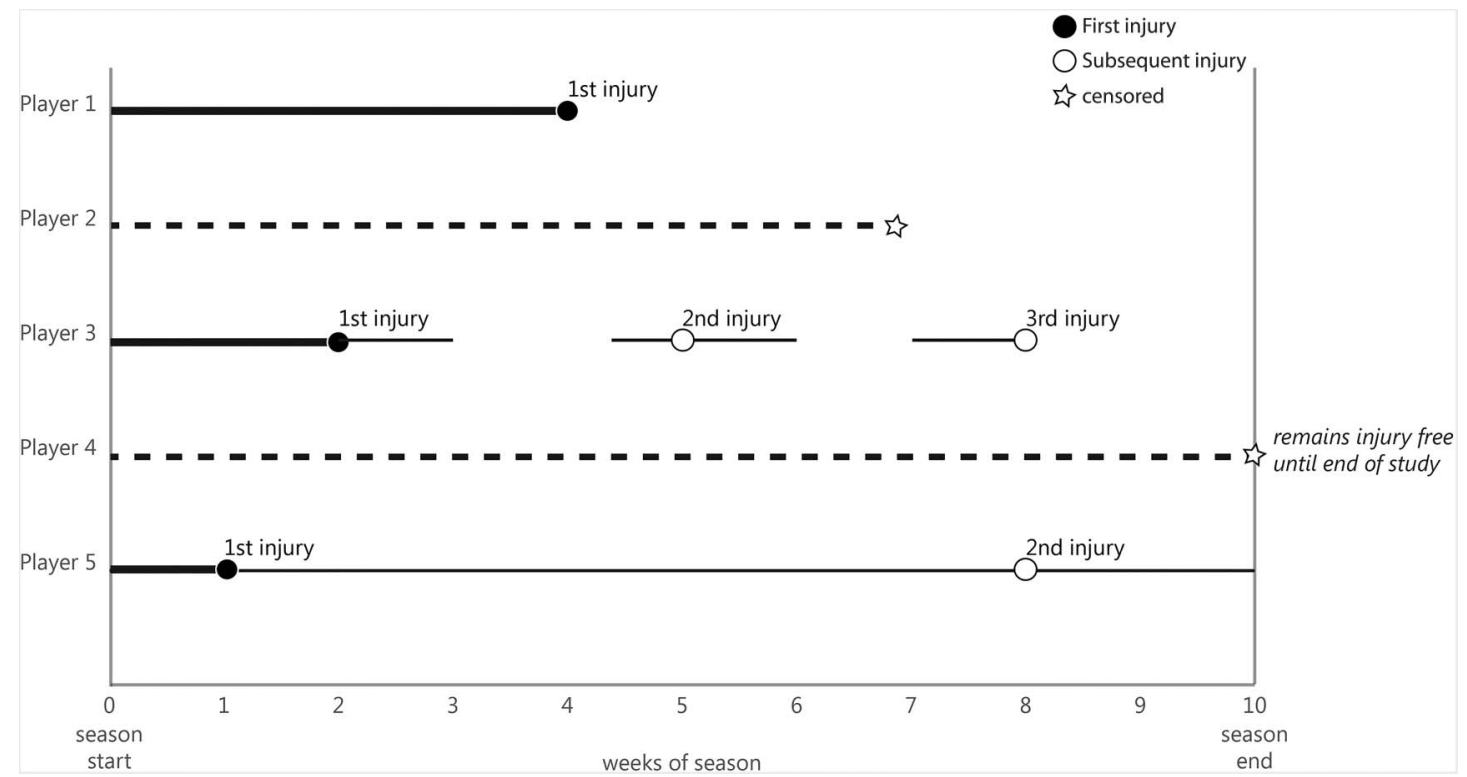

Figure 4 Common approach to reporting injury incidence in terms of time to first injury.

decision-making about recurrent injuries is lacking because the appropriate statistical techniques for subsequent injuries are currently underutilised. ${ }^{9}$ Therefore, it is recommended that sports injury epidemiologists use the SIC to fully make use of all relevant longitudinal sports injury data (as shown in figure 5).

Twitter Follow Caroline Finch at @CarolineFinch

Acknowledgements This material was presented by Caroline F Finch as part of a conference presentation at the Be Active 2014 Conference organised in Canberra, Australia by Sports Medicine Australia. She was awarded the ASICS Best Paper Injury Prevention award for her presentation at this conference.

Funding CFF is funded by a National Health and Medical Research Council (NHMRC) Principal Research Fellowship (ID1058737). ACRISP is one of the International Research Centres for the Prevention of Injury and Protection of Athlete Health supported by the IOC. The University of North Carolina Injury Prevention Research Center is partially supported by an award R49CE002479 from the National Center for Injury Prevention and Control, Centers for Disease Control and Prevention.

Competing interests None declared.

Provenance and peer review Not commissioned; externally peer reviewed. 


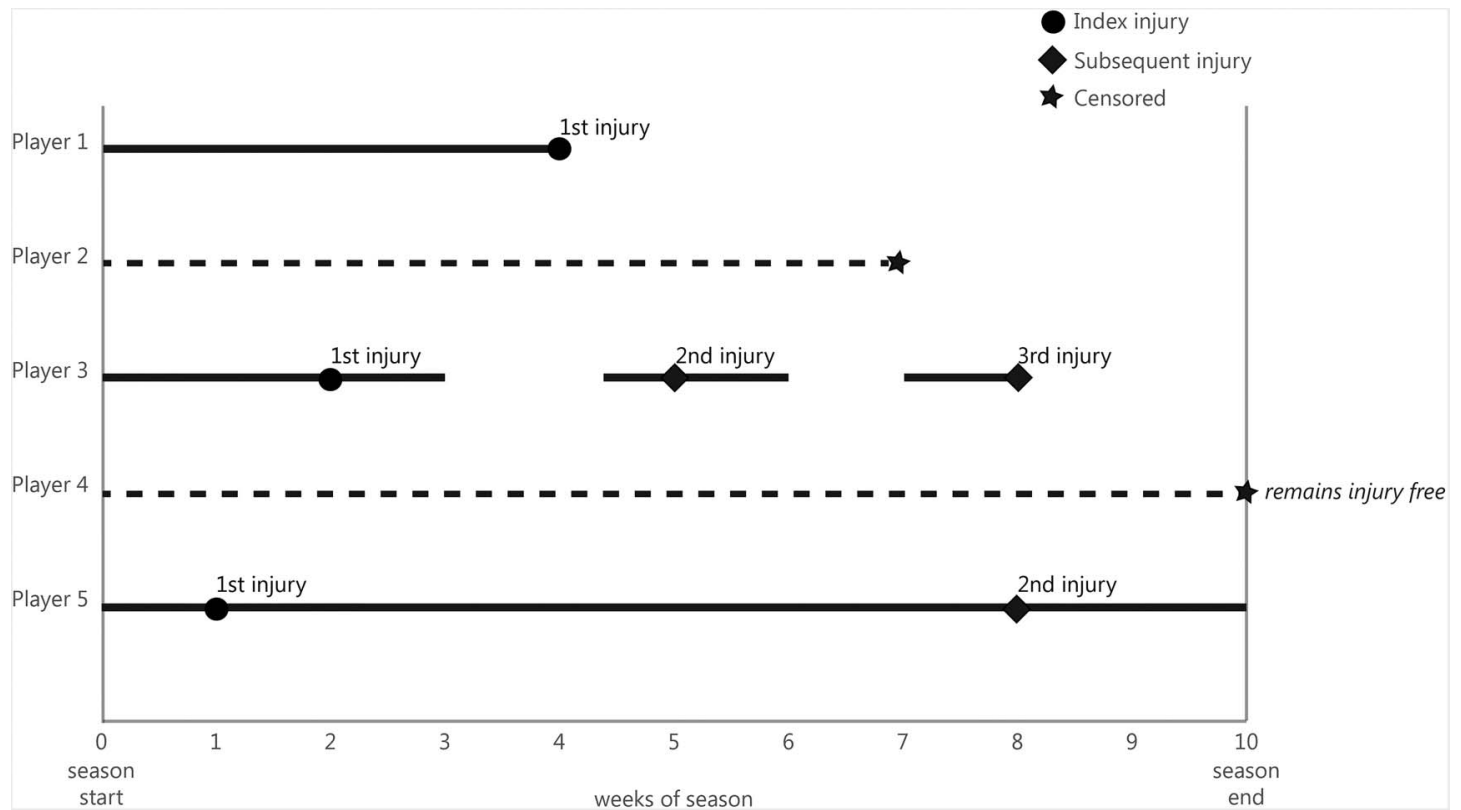

Figure 5 Illustration of relationships between index and subsequent injury.

original work is properly cited and the use is noncommercial. See: http://creativecommons.org/licenses/ by-nc/4.0/

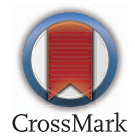

To cite Finch $C_{F}$, Marshall SW. Br J Sports Med 2016;50:712-715.

Accepted 1 September 2015

Published Online First 21 September 2015

Br J Sports Med 2016:50:712-715.

doi:10.1136/bjsports-2015-094719

\section{REFERENCES}

1 Engebretsen L, Bahr R, Cook JL, et al. The IOC Centres of Excellence bring prevention to sports medicine. Br J Sports Med 2014:48:1270-5.

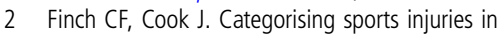
epidemiological studies: the subsequent injury categorisation $(\mathrm{SIC})$ model to address multiple, recurrent, exacerbation or new injuries. $\mathrm{Br}$ I Sports Med 2014;48:1276-80.

3 Ullah S, Gabbett TJ, Finch CF. Statistical modelling for recurrent events: an application to sports injuries. $\mathrm{Br} J$ Sports Med 2014;48:1287-93.

4 Hagglund $M$, Walden M, Ekstrand J. Previous injury as a risk factor for injury in elite football: a prospective study over two consecutive seasons. $\mathrm{Br}$ I Sports Med 2006;40:767-72.
5 Hamilton GM, Meeuwisse WH, Emery CA, et al. Subsequent injury definition, classification and consequence. Clin I Sport Med 2011;21:508-14.

6 Shrier I, Steele RJ, Hanley J, et al. Analyses of injury count data: Some do's and don'ts. Am J Epidemiol 2009;170:1307-15.

7 Timpka T, Jacobsson J, Bickenbach J, et al. What is a sports injury? Sports Med 2014;44: 423-8.

8 Hamilton G, Shrier I, Emery CA, et al. Past injury as a risk factor: an illustrative example where appearances are deceiving. Am J Epidemiol 2011;173:941-8

9 Mahmood A, Ullah S, Finch C. Application of survival models in sports injury prevention research: a systematic review. Br J Sports Med 2014;48:630. 